\title{
INFLUÊNCIA DA ADIÇÃO DE CELULOSE NANOFIBRILADA (CNF) NAS PROPRIEDADES ÓPTICAS E FÍSICAS DO PAPEL
}

\author{
INFLUENCE OF NANOFIBRILLATED CELLULOSE (NFC) ON OPTICAL AND PHYSICAL PAPER \\ PROPERTIES
}

\author{
Daniele Cristina Potulski ${ }^{1}$, Marina Stygar Lopes ${ }^{2}$, Graciela Inês Bolzón de Muniz ${ }^{3}$, Mayara Elita \\ Carneiro ${ }^{4}$, Alan Sulato de Andrade 5
}

1, 2, 3, 4, 5 Universidade Federal do Paraná, Curitiba, Paraná, Brasil-danielepotulski@gmail.com, marinastygar@gmail.com,gbmunize@ufpr.br, mayaraecarneiro@gmail.com \&alansulato@gmail.com

\section{RESUMO}

O objetivo deste trabalho foi avaliar a influência da incorporação de celulose nanofibrilada (CNF) sobre as propriedades ópticas e físicas do papel. Foi utilizada polpa Kraft branqueada de Eucalyptus sp. para produção das folhas. Para produção da CNF, a polpa foi processada mecanicamente no moinho desfibrilador Super Masscolloider Masuko Sangyo. Para visualização das estruturas e dimensões da celulose e da CNF, foi usada a microscopia eletrônica de transmissão e de varredura. A viscosidade foi usada para verificar o grau de polimerização das amostras. $O$ índice de cristalinidade foi obtido usando difração de Raios-X. Foram confeccionados papéis com grau de refinação 15 e $25{ }^{\circ} \mathrm{SR}$, com adição de $0 \%$ a $6 \%$ de CNF. Na sequência, estes foram avaliados por meio das propriedades ópticas (alvura e opacidade) e propriedades físicas (espessura, densidade aparente e permeância ao ar). O processo mecânico gerou o desfibrilamento interno e externo, promovendo a redução nas dimensões das fibras para a escala nanométrica. O processo de desfibrilação também causou a redução da cristalinidade e da viscosidade. De forma geral, as pequenas variações observadas para a alvura e a opacidade, bem como os aumentos significativos na densidade aparente e na resistência à passagem ao ar possibilitam a utilização da celulose nanofibrilada como um material renovável e biodegradável na produção de papéis.

PALAVRAS-CHAVE: Aditivos para papel, Eucalyptus, Nanofibrilas, Nanotecnologia.

\section{ABSTRACT}

The objective of this research was to evaluate the influence of nanofibrillated cellulose on the optical and physical properties of paper. Delignified Eucalyptus $s p$. Kraft pulp was used to produce paper. To produce NFC, the pulp was processed in a Super Massocolloider Masuko Sangyo grinder. To visualize the structures and dimensions of cellulose and NFC, both transmission and scanning electron microscopy were used. The viscosity was used to verify the degree of polymerization of the samples. The crystallinity index of cellulose and NFC in the films was obtained by X-ray diffraction. Paper was prouced with degree of refining at 15 and 25 @SR, with an addition of NFC ranging from 0 to $6 \%$. This process was assessed through both optical (whiteness and opacity) and physical (thickness, apparent density, and air resistance) properties. The mechanical process generates internal and external defibrillation, thus providing a size reduction of fibers to nanometric dimensions. The defibrillation process also caused reduction of cristallinity and viscosity. In general, the small variations noted for the whiteness and opacity, as well as significant increase in apparent density and air resistance, enable the use of this renewable and biodegradable material in paper production.

KEYWORDS: Eucalyptus, Nanofibrils, Nanotechnology, Paper additives. 


\section{INTRODUÇÃO}

Muitos são os materiais produzidos a partir de recursos não renováveis que têm sido aplicados como aditivos para papel a fim de promover melhorias em sua qualidade. A utilização de tais materiais acarreta uma série de inconvenientes, incluindo questões ambientais, além de altos custos.

Por isso novas pesquisas buscam a utilização de matérias-primas renováveis e biodegradáveis, de menor impacto ambiental e que possuam propriedades específicas, entre elas características de promover o aumento da resistência do papel, mantendo suas propriedades físicas e ópticas.

A nanocelulose ou celulose nanofibrilada (CNF) produzida a partir das próprias fibras de celulose da madeira, por meio do processo de desfibrilação mecânica possuem propriedades, tais como a área de superfície elevada, baixa densidade, alta capacidade de formar ligação de hidrogênio intra e intermoleculares, e alta resistência mecânica, além de ser produzida a partir de um recurso renovável e biodegradável, a celulose (NOGI et al., 2009; SIRÓ \& PLACKETT, 2010; BELBEKHOUCHE et al., 2011).

A CNF obtida por processos nanotecnológicos pode atingir diâmetros inferiores a $100 \mathrm{~nm}$ e comprimentos de vários micrômetros (STELTE \& SANADI, 2009; SEHAQUI et al., 2011; SYVERUD et al., 2011). Entre os diversos métodos de obtenção da CNF, o processo de micro moagem ou desfibrilação mecânica por meio de um moinho é sugerido por diversos autores. (ABE \& YANO 2010; PANTHAPULAKKAL \& SAIN, 2012; WANG et al., 2013). A partir desse processo, a celulose pode ser reduzida a dimensões muito pequenas, atingindo de 25 a $100 \mathrm{~nm}$ (SEHAQUI et al. 2011). As características singulares da CNF permitem que esse material possa ser aplicado como revestimentos de papéis, na produção de filmes e papéis especiais e como aditivos na produção de papel (WANG \& SAIN, 2007).

Papéis produzidos com CNF apresentam maior densidade, flexibilidade e propriedades mecânicas de tração e arrebentamento, além da baixa porosidade que dificulta a passagem de oxigênio pelo papel, e proporciona à embalagem maior resistência (NOGI \& YANO, 2008; NOGI \& YANO, 2009; GONZÁLEZ et al., 2012). Além dos aumentos de resistência física e mecânica, a sua utilização promove melhorias na qualidade de impressão, devido à estrutura mais fechada das fibras do papel promovidas pelas ligações inter e intracelulares entre a celulose e a CNF, quando esta é utilizada como revestimento ou no acabamento superficial do papel (LUU et al., 2011a; LUU et al., 2011b).

Além de ser utilizada no setor florestal, a CNF pode ser utilizada de diversas formas, como reforço em compósitos, fármacos e cosméticos, produtos para construção, componentes de alimentos e embalagens, indústria eletrônica e elétrica, setor têxtil e biomedicina (IOELOVICH, 2008).

Dentro desse contexto, este trabalho teve por objetivo avaliar a influência da incorporação de CNF, sobre as propriedades ópticas e físicas do papel em dois diferentes graus de refino.

\section{MATERIAL E MÉTODOS}

O material utilizado neste estudo foi polpa celulósica de Eucalyptus spp. comercial e branqueada com grau de refino 15요 (Schopper-Riegler). A polpa celulósica chegou, com umidade de $11 \%$ e foi desagregada a uma consistência de $1 \%$ em desfibrador de disco tipo Bauer.

\section{Obtenção da CNF}

A partir da polpa celulósica branqueada (15 oSR) foi obtida a CNF, a $0,5 \%$ de consistência, pelo processo de desfibrilação mecânica com o moinho Microprocessador Super Masscolloider Masuko Sangyo, utilizando 30 passes no moinho e rotação de 1500 rpm.

\section{Caracterização da CNF}

A caracterização da CNF foi realizada por meio da análise de microscopia eletrônica para visualização das dimensões de suas estruturas fibrilares e realização de uma comparação com a celulose antes do processo mecânico de desfibrilação.

Para verificar se realmente houve modificação estrutural da celulose permitindo a sua transformação em CNF foi determinado o índice de cristalinidade e a viscosidade da celulose e da CNF.

\section{Caracterização Microscópica}

Para a caracterização da CNF foi utilizada a técnica de microscopia eletrônica de transmissão (MET), no Centro de Microscopia Eletrônica da Universidade Federal do Paraná (CME-UFPR), com auxílio de um microscópio eletrônico de transmissão da marca Joel, modelo JEM 1200EXII Electron Microscope (600 mil X). 
Devido à dimensão e as características da CNF as imagens foram obtidas por meio da deposição de uma gota da solução de $0,05 \%$ de CNF e água em uma tela de parlódio e a secagem foi feita naturalmente em temperatura ambiente, formando um filme muito fino, não visível a olho nu.

As fibras de celulose foram caracterizadas utilizando a técnica de microscopia eletrônica de varredura, com auxílio de um microscópio eletrônico de varredura - MEV de Bancada - Modelo FEI Phenom Tabletop Microscope do CME-UFPR. As imagens foram obtidas a partir de uma folha de papel de celulose de $60 \mathrm{~g} / \mathrm{m}^{2}$.

\section{Viscosidade}

Para avaliar o efeito do processo de desfibrilação mecânica sobre o grau de polimerização e a degradação das cadeias de celulose, realizou-se ensaio de viscosidade com as amostras de celulose e de CNF. A viscosidade foi determinada em solução de cuproetilenodiamina (CED) por meio de um viscosímetro capilar de acordo com a Norma TAPPI T 230 om-94.

\section{Índice de Cristalinidade}

Para determinação da estrutura cristalina da celulose e da CNF foi usado o difratômetro de Raios-X da Shimadzu modelo XRD-7000 juntamente com software XRD$6100 / 7000 \vee 5.0$. A velocidade de varredura adotada foi de 1 \%/min variando de 3 a 45 ㅇ, utilizando radiação de $\mathrm{Cu}$ $\mathrm{K} \alpha$ com comprimento de onda de $0,15418 \mathrm{~nm}$ e voltagem de $40 \mathrm{kV}$ com corrente de $20 \mathrm{~mA}$.

A determinação da cristalinidade foi feita em um filme $\left(60 \mathrm{~g} / \mathrm{m}^{2}\right)$ de CNF produzido a partir da deposição da solução de CNF e água em uma tela, com auxílio de uma bomba de vácuo para retirada da água e posteriormente um secador de papel (temperatura de $60^{\circ} \mathrm{C}$ ) para secagem completa do filme.

O método adotado para determinação dos índices de cristalinidade foi o sugerido por Segal et al., (1959), sendo que para cada tratamento foram realizadas duas repetições, totalizando 6 valores de índice de cristalinidade.

\section{Processo de Refinação}

A partir da polpa celulósica branqueada (15 oSR) foi separada uma parte do material que foi refinado até atingir $25^{\circ} \mathrm{SR}$ de refino. $\mathrm{O}$ processo de refinação da polpa foi feito em moinho Jokro, seguindo a norma ISO 5264/3, a uma rotação de 1500 rpm e consistência de 6\%.

O grau de refinação da polpa celulósica sem refino (15 ${ }^{\circ} \mathrm{SR}$ ) e refinada ( $25^{\circ} \mathrm{SR}$ ) foi determinado seguindo a norma ISO 5267/1, por meio do equipamento Schopper Riegler.

\section{Confecção das Folhas com Adição de CNF}

As folhas foram formadas com a polpa celulósica com $15^{\circ} \mathrm{SR}$ (polpa que não foi refinada) e polpa celulósica refinada a $25^{\circ} \mathrm{SR}$, com incorporações de CNF (dispersa em água a $0,5 \%$ de consistência) nas proporções de 0 a $6 \%$ para cada grau de refino, totalizando 14 tratamentos. Estabeleceu-se a gramatura final desejada para os filmes de $60 \pm 5 \mathrm{~g} / \mathrm{m}^{2}$.

A confecção das folhas de papel foi realizada seguindo as normas ISO 5269/2 e T205 sp-02, em uma estação formadora tipo Rapid-Köethen.

As folhas de papel foram climatizadas à temperatura de $23 \pm 2{ }^{\circ} \mathrm{C}$ e umidade relativa do ar de $50 \pm 2 \%$ para caracterização e realização dos ensaios.

\section{Ensaios Ópticos e Físicos}

As propriedades do papel avaliadas foram: propriedades ópticas (alvura - T452-om02 e opacidade T429-om01) e propriedades físicas (gramatura - T410om02, espessura - T411-om97, densidade aparente T220-om01 e Permeância ao Ar (método Gurley) - T460om02).

Os ensaios foram realizados em folhas formadas de celulose com 15 -SR e adições de CNF de 0 a $6 \%$, sendo $0 \%$ o tratamento testemunha formado apenas por folhas com polpa celulósica de 15 요 sem adição de CNF. E o mesmo foi realizado em folhas formadas com celulose de 25으 e adições de CNF de 0 a $6 \%$, sendo $0 \%$ o tratamento testemunha, sem adição de CNF, apenas o tratamento de refino.

\section{Análise Estatística}

Para verificar a influência do tratamento de desfibrilação da celulose na obtenção da CNF os valores obtidos, para o índice de cristalinidade e para a viscosidade da celulose e das nanofibrilas de celulose, foram submetidos à análise de variância com comparação de médias pelo Teste de Tukey a $5 \%$ de probabilidade. Anteriormente, foi realizado o Teste de Grubb para identificar os outliers, e o teste de normalidade. O Teste de Bartlett foi realizado para testar a homogeneidade das 
variâncias. O software utilizado foi o STATGRAPHICS Centurion $\mathrm{XVI}{ }^{\circledR}$.

A fim de avaliar se houve influência significativa da porcentagem de adição de CNF, os valores obtidos para as propriedades físicas e ópticas dos papéis com 15 e $25^{\circ} \mathrm{SR}$ foram sujeitos à análise de variância com comparação de médias pelo Teste de Tukey a $5 \%$ de probabilidade utilizando o software STATGRAPHICS Centurion XVI ${ }^{\circledR}$. O Teste de Grubb foi aplicado para identificar os outliers. Em seguida foi feito o teste de normalidade e para testar a homogeneidade das variâncias foi realizado o Teste de Bartlett.

\section{RESULTADOS E DISCUSSÃO}

\section{Caracterização Microscópica}

As fibras de celulose antes de receberam algum tipo de tratamento mecânico apresentam diâmetro micrométrico e pouca fibrilação da parede celular, conforme demonstrado nas Figuras 1 (A e B).

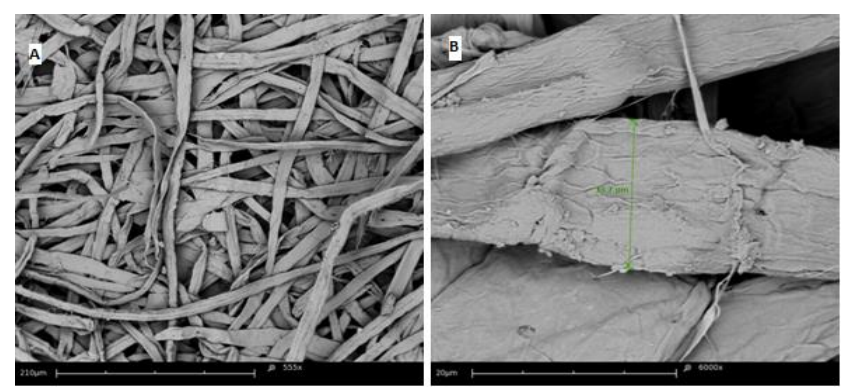

Figura 1. Celulose de Eucalyptus sp. em diferentes escalas.

As nanofibrilas de celulose obtidas após 30 passes pelo moinho desfibrilador estão apresentadas nas Figuras 2(A e B), na qual é possível observar que o processo de desfibrilação mecânica provocou a fibrilação da parede celular das fibras de celulose, reduzindo o seu diâmetro e gerando as nanofibrilas, elementos que apresentam pelo menos uma de suas dimensões menores que $100 \mathrm{~nm}$ (STELTE \& SANADI, 2009; QUA \& HORNSBY, 2011).

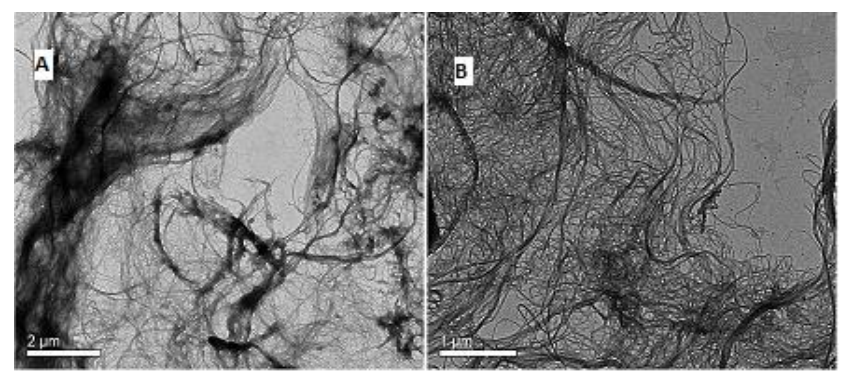

Figura 2. CNF (Celulose nanofibrilada) em diferentes escalas.
Nota-se redução significativa no diâmetro das fibras com o processo de desfibrilação mecânica no moinho após 30 passes. A largura das fibras reduziu de dimensões micrométricas para dimensões nanométricas.

\section{Viscosidade e Índice de Cristalinidade}

O processo de desfibrilação mecânica reduziu de forma expressiva a dimensão das cadeias de celulose ou alterou de alguma forma a sua configuração, consequentemente reduzindo seu grau de polimerização.

Com base na Tabela 1 é possível observar a diferença significativa entre o grau de polimerização da celulose (17,3 mPa.s) e da CNF (6,8 mPa.s).

Tabela 1. Viscosidade e Índice de Cristalinidade da Celulose e da CNF

\begin{tabular}{ccc}
\hline Tratamento & Viscosidade (mPa.s) & $\begin{array}{c}\text { Índice de } \\
\text { Cristalinidade (\%) }\end{array}$ \\
\hline Celulose & $17,3(\mathrm{a}) \pm 0,1$ & $81,5(\mathrm{a}) \pm 0,2$ \\
CNF & 6,8 (b) $\pm 0,1$ & $67,1(\mathrm{~b}) \pm 1,0$ \\
\hline
\end{tabular}

Em que: Médias e desvio padrão. Médias seguidas de letras diferentes diferem entre si pelo teste Tukey, com um nível de significância de $5 \%$.

Milanez et al. (2008) encontraram valores de viscosidade de $18,7 \mathrm{mPa} .5$ para polpa Kraft branqueada de Eucalyptus, valores próximos aos obtidos neste estudo para celulose Kraft branqueada de Eucalyptus antes do processo de desfibrilação.

A diferença entre a viscosidade da celulose e da CNF também foi verificada em outros estudos. Pöhler et al. (2010) observou em um estudo sobre a influência dos métodos de desfibrilação nas características das nanofibrilas de celulose que a viscosidade média da celulose é superior a viscosidade média da CNF obtida pelo método de desfibrilação mecânica por meio do moinho Super Masscolloider Masuko Sangyo. Zimmermann et al. (2010) também verificaram redução entre $15 \%$ e $63 \%$ na viscosidade devido à desfibrilação mecânica, em um estudo sobre as propriedades de nanofibrilas de celulose de diferentes matérias primas.

0 índice médio de cristalinidade da celulose foi de $81,52 \%$ e da CNF de $67,13 \%$ (Tabela 1). Observa-se que a celulose apresenta índice de cristalinidade estatisticamente superior ao índice de cristalinidade da CNF. Isso normalmente ocorre, pois o processo de desfibrilação mecânica promove alterações irreversíveis nas fibras, devido aos fenômenos de fibrilação interna e externa. 
A região amorfa menos ordenada e por isso menos resistente se torna o ponto frágil no processo de desfibrilação, no qual se inicia o desenrolamento das fibrilas até seu completo arrancamento, que pode atingir a região cristalina. $O$ desfibrilamento interno e externo da fibra além de causar a redução da cristalinidade e da viscosidade gera redução nas dimensões das fibras, que após o tratamento mecânico passam a apresentar-se em escala nanométrica.

O valor médio do índice de cristalinidade da celulose obtido neste estudo está próximo ao observado por Lengowski et al. (2013), em um estudo sobre a avaliação de métodos de obtenção de celulose com diferentes graus de cristalinidade, no qual o índice de cristalinidade da celulose Kraft de Eucalyptus spp. foi de 79,22\%.

O grau de cristalinidade da celulose refere-se à quantidade relativa de celulose na região cristalina, na qual a fibra apresenta maior resistência à tração e ao alongamento, sendo de grande importância, pois influencia diretamente nas propriedades mecânicas do papel ou filme (FENGEL \& WEGENER, 1984). A determinação do índice de cristalinidade, por meio do grau de cristalinidade, permite analisar a possível degradação sofrida pela celulose na fibra resultante do processo mecânico de desfibrilação no moinho.

A Figura 3 apresenta as intensidades dos picos cristalinos localizados entre os ângulos de $22 \circ \leq 2 \theta \leq 23$ ㅇ e do halo de regiões de domínios cristalográficos contidos em uma região amorfa ( $18^{\circ} \leq 2 \theta \leq 19$ ㅇ) para a celulose e para a CNF.

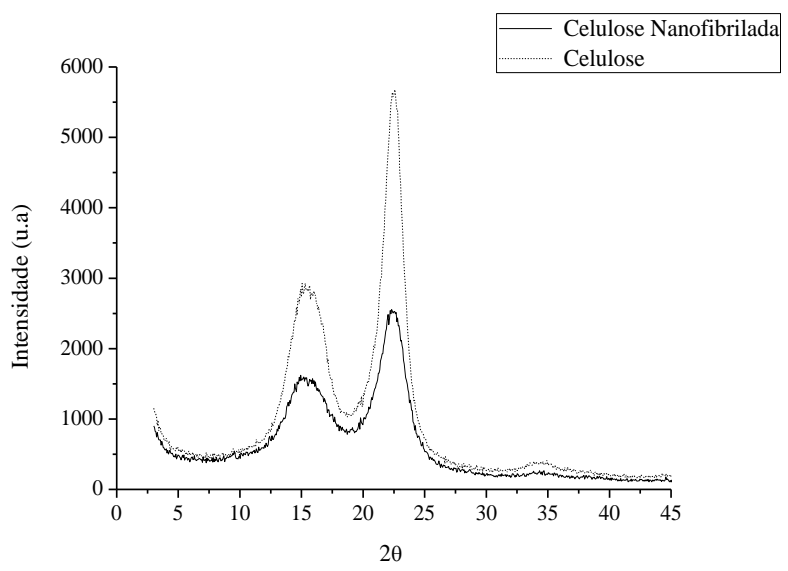

Figura 3. Difratograma de Raios-X da CNF e da celulose.

A diferença entre os picos cristalinos da celulose e da CNF é justificada em estudos recentes por autores que relatam que o tratamento mecânico em moinho desfibrilador pode provocar a nanofibrilação da celulose e também promover a degradação da mesma, fato que é percebido pela redução nos valores do grau de cristalinidade e polimerização com o aumento da intensidade do processo de desfibrilação (IWAMOTO et al., 2007; IWAMOTO et al., 2008).

\section{Ensaios Ópticos e Físicos}

As propriedades ópticas são indicadores da qualidade do papel e de polpas celulósicas. Sendo a alvura relacionada ao grau de deslignificação do papel e a opacidade ao coeficiente de dispersão de luz.

Os valores médios das propriedades ópticas podem ser observados na Figura 4.
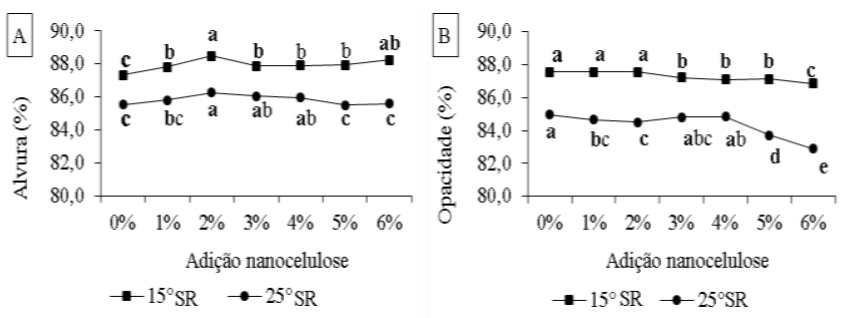

Figura 4. Valores médios Propriedade Ópticas com a adição de diferentes quantidades de CNF. A) Alvura; B) Opacidade.

Em que: Médias seguidas de letras diferentes na mesma linha do gráfico diferem entre si pelo teste Tukey, com um nível de significância de $5 \%$.

Os valores médios de alvura variaram de 87,34 a $88,48 \%$ e 85,53 a $86,25 \%$ para os tratamentos com adição de CNF sem refino e com grau de refino $25^{\circ} \mathrm{SR}$, respectivamente (Figura $4 \mathrm{~A}$ ).

A diferença estatística observada com a redução da alvura entre os tratamentos com a adição de CNF pode ter sido causada devido à distribuição das fibras e da CNF ao papel, sendo que em algumas regiões provavelmente as fibras e a CNF ficaram aglomeradas. Isso pode ocorrer durante a formação da folha devido às características da CNF. Com relação ao refino, este promove a redução da alvura devido ao melhor entrelaçamento das fibras resultado do tratamento mecânico deste processo sobre as fibras de celulose.

A opacidade apresentou valores médios que variaram de 86,86 a $87,55 \%$ e 82,90 a $84,97 \%$ para os tratamentos com adição de CNF sem refino e com refino, respectivamente (Figura 4B). A diferença entre as médias foi significativa, e os valores foram superiores aos observados por Milanez et al. (2008) para papéis produzidos a partir de polpa Kraft de Eucalyptus com kappa 1,4,78,4\% para papéis produzidos com grau de refino $16,6^{\circ}$ e $70,8 \%$ para papéis produzidos com grau de 
refino 35,2. A diferença entre os valores encontrados nos trabalhos devem-se provavelmente a diferenças nos valores de grau de refino e características das fibras de celulose e do processo de branqueamento.

González et al. (2012) estudaram as propriedades ópticas, físicas e mecânicas de papéis de Eucalyptus com diferentes graus de refino e com adição 0; 3; e $6 \%$ de CNF, e observaram diferença nos resultados de opacidade para os diferentes graus de refino como observado neste estudo, mas não encontraram diferenças na opacidade com a adição de CNF.

A opacidade tende a diminuir em papéis produzidos com fibras refinadas, por isso, observa-se uma redução significativa para os tratamentos com grau de refino $25^{\circ} \mathrm{SR}$, especialmente, com adição de 5 e $6 \%$ de CNF, nos quais a redução da propriedade foi mais significativa. Isso porque o aumento de CNF na composição do papel causa a redução do coeficiente de dispersão de luz que está relacionado com a estrutura das folhas e distribuição das fibras e da CNF no papel, pois a CNF devido a sua pequena dimensão ocupa os pequenos espaços vazios na estrutura do papel, tornando a trama de fibras no papel mais fechada e consequentemente menos transparente.

De forma geral a adição de CNF gerou a redução de aproximadamente $3 \%$ na alvura do papel, enquanto que a opacidade diminui em torno de $6 \%$ com a adição de CNF. Sendo assim é possível verificar que as propriedades ópticas, de forma prática, tiveram sua qualidade ligeiramente reduzida com a adição de CNF, permitindo assim a sua utilização para aumento das propriedades mecânicas que já foram verificadas em outros estudos (POTULSKI et al., 2013).

A Figura 5 apresenta os valores médios das propriedades físicas (espessura, densidade aparente e permeância a passagem do ar) do papel com adição de CNF não refinado e refinado.

Os valores de gramatura variaram de 61,62 a 64,20 $\mathrm{g} / \mathrm{m}^{2}$ para os tratamentos com adição de CNF aos papéis sem tratamento de refino, e de 59,93 a $64,48 \mathrm{~g} / \mathrm{m}^{2}$ para os tratamentos com adição de CNF aos papéis de grau de refino $25^{\circ} \mathrm{SR}$, e estão dentro da variação proposta no trabalho.

A espessura variou de 132,80 a 146,60 $\mu \mathrm{m}$ e de 106,60 a $122,20 \mu \mathrm{m}$, para os tratamentos com adição de CNF sem refino e com refino, respectivamente (Figura 5A). Os valores apresentaram diferença estatística entre si e tendência de redução com a adição de CNF e com o tratamento de refino.

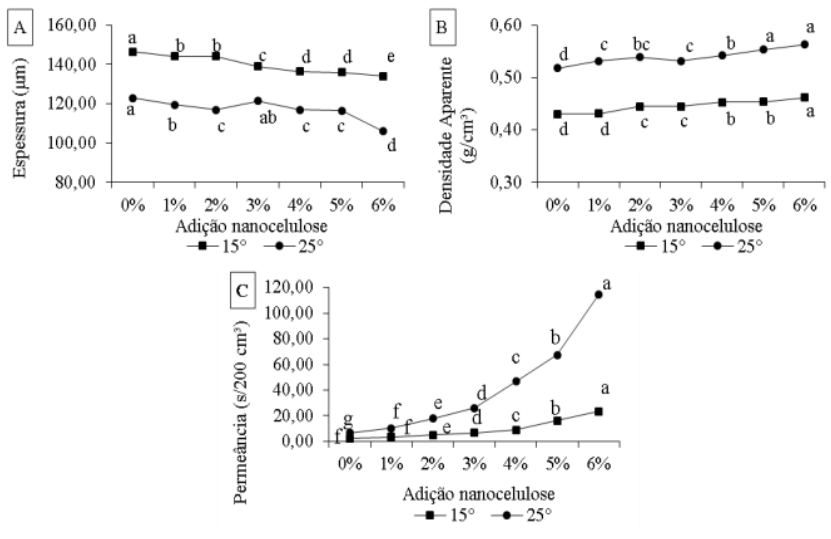

Figura 5. Valores médios propriedade físicas. A) Espessura; B) Densidade Aparente; C) Permeância ao ar (Gurley) com a adição de diferentes quantidades de CNF.

Em que: Médias seguidas de letras diferentes na mesma linha do gráfico diferem entre si pelo teste Tukey, com um nível de significância de $5 \%$.

Os tratamentos de desfibrilação mecânica promovem ao papel maior quantidade de ligações intra e intermoleculares gerando um melhor arranjo das fibras levando a formação de papéis mais uniformes e com estrutura mais compacta, consequentemente papéis de menor espessura.

A densidade aparente, para os papéis pertencentes ao tratamento com adição de CNF sem refino e com refino variou de 0,43 a $0,46 \mathrm{~g} / \mathrm{m}^{3}$ e 0,52 a $0,56 \mathrm{~g} / \mathrm{m}^{3}$, respectivamente (Figura $5 \mathrm{~B}$ ). Os valores apresentaram diferentes estatisticamente entre si, sendo que para graus de refino observa-se tendência crescente com o aumento da adição da CNF.

O aumento da densidade aparente, bem como, a redução da espessura, está relacionado com a estrutura mais compacta dos papéis resultante dos processos de desfibrilação mecânica.

Os valores médios de permeância ao ar - método Gurley variaram de 2,40 a $24,07 \mathrm{~s} / 200 \mathrm{~cm}^{3}$ e 6,72 a 111,40 $\mathrm{s} / 200 \mathrm{~cm}^{3}$ para os papéis pertencentes ao tratamento com adição de CNF sem refino e com refino, respectivamente (Figura 5C). Observa-se que a resistência à passagem ao ar aumenta com a adição da CNF, isso devido ao aumento da densidade do papel e a baixa porosidade, consequência da desfibrilação mecânica das fibras.

A permeância ao ar pode ser utilizada para estimar a resistência à passagem de outros fluídos, como a resistência a passagem de oxigênio através da embalagem e redução da penetração de tintas no papel, o que possibilitaria a utilização dos papéis com impressões frente e verso com melhor qualidade (PHILIPP \& 
ALMEIDA, 1988).

A adição de CNF tanto em composições de fibras não refinadas $\left(15^{\circ} \mathrm{SR}\right)$ assim como em de fibras refinadas $\left(25^{\circ} \mathrm{SR}\right)$ promovem melhoria em suas propriedades físicas sem afetar suas propriedades ópticas, o que possibilita a utilização da CNF como aditivo para papel.

\section{CONCLUSÕES}

As imagens obtidas por microscopia eletrônica de transmissão e microscopia eletrônica de varredura indicam que a dimensão das fibras reduziu significativamente com o processo de desfibrilação mecânica, sendo possível obter material com dimensões nanométricas.

A viscosidade e o índice de cristalinidade da CNF foram inferiores à da celulose, devido à modificação estrutural causada pelo processo de desfibrilação mecânica.

Em relação à alvura e a opacidade (propriedades ópticas), os valores médios foram estatisticamente diferentes para os tratamentos com adição de CNF. A redução máxima da alvura e da opacidade foi de $3 \%$ e $5 \%$, respectivamente.

Para as propriedades físicas verificou-se que a presença de CNF aumenta a densidade aparente e a resistência à passagem ao ar e diminui a espessura do papel. A mesma tendência foi observada tanto para os papéis não refinados quanto para os sujeitos ao tratamento de refino.

\section{REFERÊNCIAS}

ABE, K.; YANO, H. Comparison of the characteristics of cellulose microfibril aggregates isolated from fiber and parenchyma cells of Moso bamboo (Phyllostachys pubescens). Cellulose, v.17, n.2, p.271-277, 2010.

BELBEKHOUCHE, S. et al. Water sorption behavior and gas barrier properties of cellulose whiskers and microfibrils films. Carbohydrate Polymer, v.83, n.4, p.1740-1748, 2011.

FENGEL, D.; WEGENER, G. Wood chemistry, ultrastructure, reactions. New York: Walter de Gruyter, 1984.

GONZÁLEZ, I. et al. Nanofibrillated cellulose as paper additive in Eucalyptus pulps. BioResources, v.7, n.4, p.5167-5180, 2012.

IOELOVICH, M. Cellulose as a nanostructured polymer: a short review. BioResources, v.3, n.4, p.1403-1418, 2008.

IWAMOTO, S. et al. Nano-fibrillation of pulp fibers for the processing of transparent nanocomposites. Applied Physics A, v.89, n.2, p.461-466, 2007.
IWAMOTO, S. et al. The Effect of Hemicelluloses on Wood Pulp Nanofibrillation and Nanofiber Network Characteristics. Biomacromolecules, v.9, n.3, p.1022-1026, 2008.

LENGOWSKI, E.C. et al. Avaliação de métodos de obtenção de cellulose com diferentes graus de cristalinidade. Scientia Forestalis, v.41, n.98, p.185-194, 2013.

LUU, W.T. et al. Application of nano-fibrillated cellulose as a paper surface treatment for inkjet printing. PaperCon Symposium, p.2222-2233, 2011a.

LUU, W.T.; et al. Nano-fibrillated cellulose as a paper surface treatment for inkjet printing. TAPPI. International Conference on Nanotechnology for Renewable Materials, Washington, 2011b.

MILANEZ, A.F. et al. Condições ótimas para branquear polpa kraft de eucalipto com uma sequência de três estágios. Scientia Florestalis, v.36, n.78, p.105-113, 2008.

NOGI, M.; YANO, H. Transparent Nanocomposites Based on Cellulose Produced by Bacteria Offer Potential Innovation in the Electronics Device Industry. Advanced Materials, v.20, n.10, p.1849-1852, 2008.

NOGI, M.; YANO, H. Optically transparent nanofiber sheets by deposition of transparent materials: A concept for a roll-to-roll processing. Applied physics letters, v.94, p.1-4, 2009.

NOGI, M. et al. Optically transparent nanofiber paper. Advanced Materials, n.21, n. 16, p.1595-1598, 2009.

PANTHAPULAKKAL, S.; SAIN, M. Preparation and characterization of cellulose nanofibril films from wood fibre and their thermoplastic polycarbonate composites. International Journal of Polymer Science, v.2012, n.249, p.1-6, 2012.

PHILIPP, P.; ALMEIDA, M.L.O. D'. Celulose e papel: tecnologia de fabricação da pasta celulósica. 2.ed. São Paulo: IPT, 1988.

PÖHLER, T. et al. Influence of fibrillation method on the character of nanofibrillated cellulose (NFC). VTT Technical Research Centre of Finland. The Finnish Centre for Nanocellulosic Technologies Espoo, Finland, 2010.

POTULSKI, D.C. et al. The influence of incorporation of microfibrillated cellulose on mechanical strength properties of paper. Scientia Florestalis, v.42, n.103, p.345-351, 2014.

QUA, E.H.; HORNSBY, P.R. Preparation and characterization of nanocellulose reinforcedpolyamide-6. Plastics, Rubber and Composites, v.40, n.6/7, p.300-306, 2011.

SEGAL, L. et al. An empirical method for estimating the degree of crystallinity of native cellulose using the X-ray diffractometer. Textile Research Journal, v.29, n.10, p.786-794, 1959.

SEHAQUI, H. et al. Wood cellulose biocomposites with fibrous structures at micro- and nanoscale. Composites Science and Technology, n.71, n.3, p.382-387, 2011.

SIRÓ, I.; PLACKETT, D. Microfibrillated cellulose and new nanocomposites materials: A review. Cellulose, v.17, n.3, p.459494, 2010. 
STELTE, W.; SANADI, A.R. Preparation and characterization of cellulose nanofibers from two commercial hardwood and softwood pulps. Industrial \& Engineering Chemistry Research, v.48, n.24, p.11211-11219, 2009.

SYVERUD, K. et al. A comparative study of Eucalyptus and Pinus radiata pulp fibres as raw materials for production of cellulose nanofibrils. Carbohydrate Polymers, v.84, n.3, p.1033-1038, 2011.

TECHNICAL ASSOCIATION OF THE PULP AND PAPER INDUSTRY TAPPI. T205-om02. Forming handsheets for physical tests of pulp. In: Tappi Test Methods. TAPPI Press, Norcross, GA, 2004.

TECHNICAL ASSOCIATION OF THE PULP AND PAPER INDUSTRY TAPPI. T 220-om01. Physical testing of pulp handsheets. In: Tappi Test Methods. TAPPI Press, Norcross, GA, 2004.

TECHNICAL ASSOCIATION OF THE PULP AND PAPER INDUSTRY TAPPI. T230 om-94. Viscosity of pulp: capillary viscometer method. In: Tappi Test Methods. TAPPI Press, Atlanta, 1999.

TECHNICAL ASSOCIATION OF THE PULP AND PAPER INDUSTRY TAPPI. T 410-om02. Grammage of paper and paperboard. In: Tappi Test Methods. TAPPI Press, Norcross, GA, 2004.

TECHNICAL ASSOCIATION OF THE PULP AND PAPER INDUSTRY TAPPI. T 411-om97. Thickness of paper, paperboard, and combined board. In: Tappi Test Methods. TAPPI Press, Norcross, GA, 2004.

TECHNICAL ASSOCIATION OF THE PULP AND PAPER INDUSTRYTAPPI. T460 om-02. Air resistance of paper (Gurley method). In: Tappi Test Methods. TAPPI Press, Norcross, GA, 2004.

WANG, B.; SAIN, M. Isolation of nanofibers from soybean source and their reinforcing capability on synthetic polymers. Composites Science and Technology, v.67, p.2521-2527, 2007.

WANG, $\mathrm{H}$. et al. Preparation of Ultralong Cellulose Nanofibers and Optically Transparent Nanopapers Derived from Waste Corrugated Paper Pulp. Bioresources, v.8, n.1, p.1374-1384, 2013.

ZIMMERMANN, T. et al. Properties of nanofibrillated cellulose from different raw materials and its reinforcement potential. Carbohydrate Polymers, v.79, n.4, p.1086-1093, 2010. 